\title{
El argumento interpretativo intercultural en la Corte Constitucional
}

\section{The intercultural interpretative argument in the Constitutional Court}

Vicente Manuel Solano Paucay ${ }^{1 *}$

${ }^{1}$ Universidad Católica de Cuenca

*vsolanop@ucacue.edu.ec

DOI: https://doi.org/10.26871/killkana_social.v2i4.353

\begin{abstract}
Resumen
La Constitución del año 2008, reconoce al Ecuador como un Estado intercultural y plurinacional, al hacer explícito el reconocimiento de los derechos de las comunidades, pueblos y nacionalidades. Entonces la plena vigencia de los derechos, subyace a una interpretación de carácter intercultural que conlleva una argumentación distinta a los cánones interpretativos tradicionales. Así, la Corte Constitucional en algunas decisiones judiciales ha utilizado como "método de interpretación" lo que denomino el "argumento interpretativo intercultural". En este artículo se pretende reconstruir los elementos necesarios que usa el intérprete oficial de la Constitución para forjar este nuevo argumento interpretativo.
\end{abstract}

Palabras clave: nuevo constitucionalismo latinoamericano, neoconstitucionalismo, interculturalidad, interpretación jurídica, control de constitucionalidad.

\section{Abstract}

The Constitution of 2008 recognizes Ecuador as an intercultural and plurinational State, by making explicit the recognition of the rights of communities, peoples and nationalities. Then the full validity of the rights, underlies an intercultural interpretation that entails an argument different from the traditional interpretative canons. Thus, the Constitutional Court has used as a "method of interpretation" in some judicial decisions what I call the "intercultural interpretive argument". In this article I intend to reconstruct the necessary elements that the official interpreter of the Constitution uses to forge this new interpretive argument.

Key words: new Latin American constitutionalism, interculturalidad, neoconstitucionalismo, legal interpretation, control of constitutionality.

\section{Introducción}

El reconocimiento de los derechos de comunidades, pueblos y nacionalidades establecidos en la Constitución ecuatoriana es uno de los elementos sustanciales de lo que se comprende como el carácter intercultural y plurinacional del Estado. Así, el texto constitucional ecuatoriano como el boliviano establece como un eje transversal la interculturalidad -no como límite- para la plena vigencia de los derechos. Además, este reconocimiento no solo es del ámbito estatal sino supranacional, así tanto el Convenio 169 de la OIT y en la Declaración de las Naciones Unidas sobre los Derechos de los Pueblos Indígenas, señalan la importancia de los modos de vida subalternos o no-sistémicos.

Esta categoría implicó el reconocimiento de la jurisdicción indígena, esto conlleva la coexistencia con la justicia ordinaria. A su vez las dos anteriores están bajo la revisión de la justicia constitucional. En esta perspectiva, la Corte Constitucional es el máximo intérprete de las disposiciones normativas, lo que lleva a que la interpretación jurídica de ultima ratio está atribuida a este órgano. En definitiva, la interpretación judicial con carácter intercultural está centrada en la actividad de esta alta corte de justicia constitucional.

Por ello, el objetivo general del presente trabajo es realizar una aportación a la comprensión de los argumentos jurídicos, como base de la interpretación jurídica desarrollada en Ecuador. El objetivo específico es señalar los elementos necesarios que utiliza el intérprete oficial de la Constitución al momento de atribuir significado a los enunciados normativos que comprenden los derechos de las comunidades pueblos y nacionalidades. Este trabajo lleva a la práctica las tesis escépticas sobre la interpretación del 
derecho, que se vienen sosteniendo desde autores como G. Tarello, R. Guastini en el realismo jurídico genovés.

\section{Conceptos a distinguir}

Los derechos de los pueblos indígenas han sido reconocidos ya desde la Constitución de 1998, entre los principales: identidad, propiedad, participación, educación, posesión ancestral de las tierras comunitarias, entre otros. En la Constitución actual se han establecido nuevos derechos como la consulta pre legislativa, consulta previa, educación intercultural bilingüe, reconocimiento, no discriminación, formas de organización social, conocimientos colectivos e incluso se prescribe el etnocidio. Según la clasificación de los derechos de la Constitución del Ecuador, los derechos se clasifican en siete categorías y dentro de la tercera se encuentran los derechos de comunidades, pueblos y nacionalidades (Avila, 2012).

Sobre la concepción de interculturalidad, Catherine Walsh analiza la misma en tres perspectivas: la primera relacional, que comprende el intercambio entre culturas en contextos de igualdad o desigualdad. La segunda funcional por ser adaptable a los fines del sistema hegemónico. Y, tercera la crítica -es la que asumimos- que comprende una visión contra hegemónica y disruptiva al sistema social imperante, que implica la posibilidad de la construcción de sociedades distintas a las capitalistas (Walsh, 2012).

Ahora bien, la Corte Constitucional al ser el máximo órgano de interpretación del texto constitucional ecuatoriano, es quien debe en el ejercicio de las atribuciones que se le ha conferido, utilizar los cánones de interpretación necesarios. Bajo esta premisa la actividad interpretativa que debe desarrollar la alta Corte de Justicia Constitucional puede ser examinada a la luz de las teorías de la interpretación jurídica. En este sentido, se puede señalar que taxativamente se encentran tres principales: cognoscitivistas, intermedias y escépticas.

Las primeras indican que la labor interpretativa consiste "en conocer un significado objetivo y preexistente" (Martínez, 2010, p.49). Por tanto, el objetivo del intérprete es descubrir el significado real, verdadero de una disposición normativa. Esta perspectiva teórica, está íntimamente ligada tanto al formalismo jurídico -concepción del derecho- como al esencialismo lingüístico -concepción del lenguaje-. En definitiva esta teoría sostiene que la tarea interpretativa es un acto de conocimiento, mas no de voluntad. Las segundas, que sostiene que la interpretación puede ser un acto de conocimiento como uno de voluntad, en este sentido, bajo el convencionalismo lingüístico, se puede denotar que hay núcleos de certeza de significados. Además a una tendiente concepción "hartiana" del derecho. En consecuencia, se está frente a casos fáciles y difíciles. (Martínez, 2010)

Las terceras consisten en que el intérprete es quien debe atribuir el significado al texto. Se infiere entonces que no hay una respuesta correcta o un sentido verdadero. Este enfoque teórico está unido al convencionalismo jurídico y a la tesis de la radical indeterminación del derecho, lo que llevaría, a una posible discrecionalidad absoluta de los intérpretes -realismo jurídico-. En conclusión, la interpretación es acto de voluntad y no de conocimiento (Martínez, 2010). Esta última es la que se asume, en especial las del realismo jurídico genovés. En el siguiente apartado se intentará explicar los elementos básicos de la interculturalidad en el ordenamiento jurídico ecuatoriano.

\section{La Interculturalidad en el marco del Estado Consti- tucional}

En el uso prevaleciente de los juristas se entendería que cuando se hace uso de la categoría jurídica "Estado Constitucional de Derecho" comprende que la Constitución es la norma suprema del ordenamiento jurídico y puede ser descrita como el tipo de Estado Derecho más utilizada en Occidente (Zeta, 2007). Una idea que se propone es que esta categoría comprendería al menos dos elementos principales, en primer lugar la constitucionalización del ordenamiento jurídico (Guastini, 2013), así como la reconstrucción de las garantías institucionales que permitan efectivizar los derechos (Pisarello, 2003).

En torno a la constitucionalización del ordenamiento jurídico, Riccardo Guastini señala que hay al menos siete condiciones de constitucionalización del ordenamiento jurídico, entre ellas: 1) Una Constitución rígida 2) La garantía jurisdiccional de la Constitución 3) La fuerza vinculante de la Constitución 4) La "sobreinterpretación” de la Constitución 5) La interpretación conforme de las leyes 6) La aplicación directa de las normas constitucionales 7) $\mathrm{La}$ influencia de la Constitución sobre las relaciones políticas (Guastini, Estudios de Teoria Constitucional, 2013). Este concepto es disímil a lo que se señala por algunos autores como neoconstitucionalismo, y lo que se entiende como el nuevo constitucionalismo latinoamericano (Solano, 2016).

Sobre el segundo elemento, se puede decir que es indispensable reconstruir un amplio sistema de garantías, en primer lugar, normativas, que implican básicamente el aseguramiento de varios tipos de garantías constitucionales entre ellas, las normativas, jurisdiccionales y sociales. Esto según Pisarello, consiste en: Las normativas, desde un punto de vista formal, la reserva de ley, como posibilidad de que solo el legislativo pueda regular materias que involucren derechos constitucionales y no en sedes administrativas. Desde un punto de vista material en cambio una "obligación negativa de respeto", es decir, el deber del legislador y de la administración de abstenerse de interferir en la libertad de acción y uso de aquellos servicios básicos que los individuos o grupos se hayan preocupado, por sí mismo, en definitiva al principio de no regresividad y el entendimiento de los derechos sociales no solo como prestaciones sino como derechos negativos que obligan a no poder prohibir el uso de recursos básicos obtenidos sin arbitrariedad.

Las jurisdiccionales en cambio que como garantías secundarias, los jueces no únicamente tutelen los clásicos derechos civiles y políticos, sino los derechos sociales, 
como modo de controlar las actuaciones legislativas lesivas incluso en el deber de no regresividad en materia de derechos sociales constitucionales. Finalmente, las garantías sociales, estas representan un deber y un derecho ciudadano, en definitiva, que una pluralidad de actores con derechos y deberes de presentar y participar directamente en la formulación y activación de las garantías constitucionales. En este sentido, dice Pisarello, ciertos derechos de autonomía como el derecho de asociación, de huelga y la desobediencia civil son un valioso instrumento de defensa y actualización de la constitución (Pisarello, 2003).

En el caso ecuatoriano, Ramiro Ávila Santamaría desarrolla la noción del Estado Constitucional de Derechos y Justicia, cuando se habla de Estado Constitucional prescribe que se estructura bajo dos elementos: 1) la constitución determina el contenido de la ley y 2) la constitución es material, orgánica y procedimental. Es material porque tiene derechos que deben ser protegidos de forma específica y relevante, y son el fin del Estado. Es orgánica porque determina los órganos que forman parte del Estado que deben garantizar esos derechos. Y finalmente procedimental porque se establecen mecanismos de participación que procuran que la discusión pública sea informada y reglada, tanto para la toma de decisiones como para la producción normativa (Ávila, 2008). Por otra parte, el Estado de Justicia dice es "[e]n suma, la invocación del Estado a la justicia no significa otra cosa que el resultado del quehacer estatal, al estar condicionado por la Constitución y los derechos en ella reconocidos, no puede sino ser una organización social y política justa" (Ávila, 2008). En torno al Estado de Derechos, en cambio sostiene "[e]l Estado de derechos nos remite a una comprensión nueva del Estado desde dos perspectivas: (1) la pluralidad jurídica y (2) la importancia de los derechos reconocidos en la Constitución para la organización del Estado" (Ávila, 2008). La pluralidad jurídica, no reconoce únicamente como fuente de derecho la ley, sino por ejemplo se incorporan como fuente: a) las sentencias de la Corte Interamericana de Derechos Humanos que son de imperativo cumplimiento en Ecuador y b) el derecho indígena que ha existido y se ha aplicado en el Ecuador. Y en torno a la centralidad de los derechos se puede establecer que el fin del Estado es el reconocimiento, promoción, garantía de los derechos constitucionalmente establecidos, en definitiva, la parte dogmática cobra protagonismo en relación a la parte orgánica.

En este sentido la Constitución de 2008 reconoce y garantiza a las comunidades, pueblos y nacionalidades indígenas, aplicar y practicar su derecho propio o consuetudinario, y como única condición establece que la justicia indígena no deberá vulnerar derechos constitucionales. De la misma forma se establece que el ejercicio de la justicia indígena se hará, con base en sus tradiciones ancestrales y su derecho propio, dentro de su ámbito territorial, con participación de las mujeres. Además, se indica que el Estado garantizará que las decisiones de la jurisdicción indígena sean respetadas por las instituciones y autoridades públicas y que estarán sujetas al control de constitucionalidad.

Finalmente, establece que la ley instituirá los mecanismos necesarios para que se de la coordinación y cooperación entre la jurisdicción indígena y la jurisdicción ordinaria. Además, se establece como una garantía del debido proceso de que nadie puede ser juzgado más de una vez por la misma causa y materia, por ello los casos resueltos por la justicia indígena deberán ser considerados para este efecto. En definitiva, se deja atrás el monismo jurídico y se reconoce la existencia del pluralismo jurídico. En consecuencia, las decisiones judiciales que versen sobre derechos de las comunidades, pueblos y nacionalidades deben ser bajo un marco -argumento- interpretativo intercultural.

\section{La interpretación jurídica (una visión escéptica)}

Sobre la interpretación jurídica, como se ha señalado se pueden observar distintas teorías, que generalmente están ligadas a las concepciones del derecho: cognoscitivistas, intermedias y escépticas (Guastini, 2007). Como se ha hecho hincapié, en el caso de las teorías escépticas, estas presuponen algunas tesis, como la indeterminación radical del derecho por razones que subyacen a los problemas del lenguaje. En consecuencia se dejan de lado las perspectivas que únicamente o se refieren a casos fáciles, o a la mixtura entre casos fáciles y difíciles, en donde se pueden encontrar autores como Maccormick o Alexy (MacCormick, 2003) (Alexy, 1997) (Atienza, 2005). Y, se señala además están conexas, a una concepción sobre el derecho: el realismo jurídico. En ese sentido, la interpretación no es más que un acto de voluntad, a lo que el intérprete debe decidir cuál es el significado que le va atribuir a un texto jurídico, cuya premisa son las convenciones de la comunidad lingüística (Guastini, 2013) (Tarello, 2013).

En ese orden de ideas, se señala que los tipos de interpretación jurídica pueden ser básicamente dos, dependiendo de: 1) el sujeto que realiza la interpretación y 2) la técnica interpretativa utilizada. En el primer caso, se está frente a la interpretación: a) auténtica, b) oficial, c) judicial, d) doctrinal y e) operativa o estratégica. En el segundo caso, encontramos la técnica interpretativa: a) literal y b) correctora, esta última puede ser restrictiva o extensiva. No obstante, también existen instrumentos argumentativos para evitar o resolver problemas del sistema jurídico, entre estos están por ejemplo los argumentos: sistemático, histórico, teleológico, psicológico, sociológico, etc.

En esa línea, según Guastini un argumento interpretativo es "la razón -el argumento, precisamente-que un intérprete ofrece para sostener una tesis interpretativa" (Guastini, Interpretar y argumentar, 2014). En cuanto a la decisión interpretativa (doctrinal o judicial), esta puede ser decisoria estándar o decisoria creadora. Pero además, en el nivel judicial la argumentación de la interpretación es parte de la justificación externa. Entonces, se puede establecer que los tipos de argumentos interpretativos son 
innumerables o al menos es difícil hacer una exhaustiva taxonomía (Guastini, 2014).

Sin embargo, para el siguiente apartado, se tomarán algunos elementos de esta visión escéptica. En primer lugar, la interpretación oficial, es decir, la que realiza la Corte Constitucional y en segundo lugar, la perspectiva de los argumentos interpretativos en el marco de la interpretación decisoria judicial. Bajo estas premisas se puede finalmente reconstruir lo que se denomina en el presente artículo el argumento interpretativo intercultural, que se trata a continuación.

\section{El argumento interpretativo intercultural}

Para poder reconstruir los elementos necesarios que permitan configurar el argumento interpretativo intercultural, se revisan tres piezas judiciales importantes en materia de derechos de comunidades, pueblos y nacionalidades. Estas son: a) el caso "La Cocha", b) el caso "Waorani" y c) el caso "Amawtay Wasi".

En el primer caso, la sentencia del caso "La Cocha" en materia constitucional con respecto a la justicia indígena, denota los principales hechos del caso se resumen en que ya realizado el juzgamiento legítimo por parte de las autoridades indígenas, el Fiscal de Cotopaxi inició la instrucción fiscal en donde se dictó la prisión preventiva de tres implicados en un asesinato, desconociendo de esta forma, la decisión tomada por el tribunal indígena y contraviniendo las normas constitucionales y de los instrumentos internacionales de derechos humanos como el convenio 169 de la OIT.

En el segundo caso, el Juzgado Segundo de lo Penal de Orellana elevó a consulta a la Corte Constitucional, a fin de que se pronuncie sobre la constitucionalidad de la norma contenida en el artículo 1 de la Ley s/n, Registro Oficial N. ${ }^{\circ}$ 578-S del 27 de abril de 2009, inserto antes del artículo 441 del Código Penal, en el que se encuentra prevista la sanción por la comisión de genocidio. La consulta se dio a partir del juicio penal por genocidio N. ${ }^{\circ} 223-2013$, seguido en contra de Tocari Coba Quimintari Orengo y otros, pertenecientes a la nacionalidad indígena waorani, por la muerte de los ancianos waoranis Ompore Omehuay y Buganey Caiga.

En el tercer caso, se señala que mediante resolución del CONESUP N.O RCP.S13.No.268.04 del 22 de julio de 2004, se dispone que a partir del 30 de noviembre del 2005 , esta Universidad limite su actividad académica solamente a la ciudad de Quito por el tiempo de 5 años. Ante ello, los señores Luis Fernando Sarango Macas y Manuel Emique Quizhpe Quizhpe, en sus calidades de Rector (e) y Procurador (e), respectivamente, de la Universidad Intercultural de las Nacionalidades y Pueblos Indígenas "AMAWTAY WASI", presentaron acción por incumplimiento, solicitando que el CONESUP, de estricto cumplimiento a lo dispuesto en el Artículo 4 de la Ley de Creación de la Universidad "AMAWTAY WASI" y en el artículo 31 de su estatuto que dispone lo contrario a la resolución jerárquicamente inferior.
En los casos sub examine, se observan algunos párrafos que delimitan ciertos elementos estructurales del argumento interpretativo intercultural:

1) La interculturalidad y la plurinacionalidad son conceptos complementarios, a su vez no se contraponen al Estado unitario, sino al Estado homogéneo con lo que se reconoce la existencia de un pluralismo jurídico (Sentencia No. 0731-10-EP, 2014, p.12).

2) Se determina que al existir un pluralismo jurídico se debe tomar en cuenta ciertos principios para resolver los problemas relacionados con pueblos ancestrales, que son los siguientes:

a) continuidad histórica

b) diversidad cultural;

c) interculturalidad;

d) interpretación intercultural (Sentencia No. 0008-09SAN-CC, 2009, p.26-28) (Sentencia N. 004-14-SCN$C C, 2014$, p.19)

Estos principios según señala la Corte Constitucional tienen el siguiente contenido:

a) El de la Continuidad Histórica: el cual plasma que los pueblos y nacionalidades indígenas, no obstante su colonización, sus secuelas estructurales están presentes con sus identidades diferenciadas del resto de las sociedades nacionales, haciendo uso de sus costumbres, culturas, normas, instituciones jurídico-políticareligiosas, nociones filosóficas e idiomas, asentados en territorios indígenas en los cuales ejercen el autogobierno comunitario.

b) El de la Diversidad Cultural: a partir del cual, la función de la ley, en este caso de las normas es la de preocuparse en considerar no solo la relación entre el Estado y la ciudadanía, sino "las identidades entre los pueblos", es decir, tomar en cuenta la presencia de los distintos pueblos indígenas, con sus instituciones, sus costumbres y sus filosofías en relación con otros pueblos no indígenas que comparten un mismo territorio nacional.

c) El de la lnterculturalidad: el cual tiene que ver con el diálogo, fundamentalmente epistémico: no se trata de un diálogo en el cual los pueblos indígenas sean los convidados de piedra: el diálogo intercultural, como lo señala Osear Guardia/a Rivera, no es otra cosa que: "el diálogo entre las diferencias epistémicas que, al existir posiciones hegemónicas, son luchas cognitivas que tienen que ver con el modo en que diferentes pueblos hacen uso de diversas formas de producir y aplicar conocimiento, para relacionarse entre sí, con otros, con la naturaleza, con el territorio, con la riqueza, con la sociedad diversa."

d) El de la Interpretación lntercultural: el cual no es otra cosa que la obligatoriedad de poner en marcha una nueva lectura, una nueva forma de interpretar las situaciones y las realidades nacionales, con un enfoque sustentado en la diversidad cultural, más aún tratándose de pueblos indígenas. 
3) En cuanto a la interpretación intercultural se indica que se toma en consideración lo señalado por parte de la Corte Constitucional de Colombia donde se explicita las siguiente reglas, en caso de conflictos entre diversos sistema de derecho:

a) A mayor conservación de usos y costumbres mayor autonomía.

b) Los derechos fundamentales constitucionales constituyen el mínimo obligatorio de convivencia para todos los particulares.

c) Las normas legales imperativas (de orden público) de la República priman sobre los usos y costumbres de las comunidades indígenas, siempre y cuando protejan directamente un valor constitucional superior al principio de diversidad étnica y cultural.

d) Los usos y costumbres de una comunidad indígena priman sobre las normas legales dispositivas (Sentencia No. 0008-09-SAN-CC, 2009) (Sentencia N. 004-14-SCN-CC, 2014).

4) Se determina que en los casos penales donde estén inmersos pueblos ancestrales se desarrolló la doctrina penal denominada error de comprensión culturalmente condicionado, que implica la falta de interiorización de la norma a causa de su cultura (Sentencia N. 004-14$S C N-C C, 2014)$.

5) Se aplica el principio pro comunitas, que implica que si en el caso sub judice tomando en consideración los principios interculturales la aplicación de la norma, podría generar una afectación al principio de diversidad étnico y cultural, y por tanto a los derechos colectivos se debe velar por una aplicación más favorable a estos últimos (Sentencia N. 004-14-SCN-CC, 2014).

Con estos elementos se pueden llegar a establecer entonces que el argumento interpretativo intercultural, contiene los siguientes elementos:

1) El reconocimiento de diversos sistemas de derecho que a prima facie serian jerárquicamente iguales.

2) La lectura de los hechos y las normas deben ser tratados a la luz de la diversidad cultural.

3) Si, y solo si, se aplica este argumento en materia penal, previa aplicación de los principios interculturales.

4) Su aplicación podría ir conexa a la aplicación del principio pro comunitas.

Finalmente, cabe señalar que las altas cortes de justicia constitucional como la boliviana y la colombiana han explicitado algunos criterios similares sobre la interpretación intercultural (Arias, 2014)(Villanueva, 2015).

\section{Conclusiones}

Se puede determinar que la interpretación jurídica en materia de los derechos de las comunidades, pueblos y nacionalidades, como parte de la condición de un Estado plurinacional e intercultural, contiene diversas exigencias no pacíficas sobre los métodos de interpretación clásicos. Así, la Corte Constitucional como máximo órgano de interpretación constitucional debe cumplir su actividad interpretativa bajo el marco de la interculturalidad.

La interpretación decisoria/oficial que realiza la Corte Constitucional como se ha observado utiliza en los casos señalados una nueva forma de argumento interpretativo. Este nuevo método no puede ser encasillado en otros a raíz de su naturaleza crítica y divergente con la interpretación clásica u homogénea, como se ha determinado.

El argumento interpretativo intercultural, utilizado -creado- por la Corte Constitucional comprende ciertos elementos que deben ser examinados al momento de la resolución de problemas jurídicos que giren en torno a los derechos de comunidades, pueblos y nacionalidades con el fin de garantizar su plena vigencia en el contexto de una sociedad culturalmente diversa.

\section{Referencias Bibliográficas}

Alexy, R. (1997). Teoria de la Argumentacion Juridica . Madrid: Centro de Estudios Constitucionales.

Arias, B. (2014). Bases de la Interpretación Intercultural en un estado plural como el boliviano. Anuario Iberoamericano de Justicia Constitucional, 45-60.

Atienza, M. (2005). Las razones del Derecho. Mexico DF: UNAM.

Ávila, R. (2008). La Constitución del 2008 en el contexto andino. Análisis desde la doctrina y el derecho comparado. Ministerio de Justicia y Derechos Humanos.

Avila, R. (2012). Los derechos y sus garantias. Quito: Corte Constitucional para el Período de Transición.

Guastini, R. (2007). Teoría e ideología de la interpretación constitucional. Trotta.

Guastini, R. (2013). El realismo jurídico redefinido. . Palestra.

Guastini, R. (2014). Interpretar y argumentar. Madrid: Centro de Estudios Políticos y Constitucionales.

MacCormick, N. (2003). Legal reasoning and Legal Theory. Oxford: Oxford University Press.

Martínez, D. (2010). Metodología Jurídica y Argumentación . Madrid: Marcial Pons.

Pisarello, G. (2003). El Estado Social como Estado Constitucional: Mejores garantias, mas democracia. Fontamara.

Sentencia N. 004-14-SCN-CC. (2014). Descargado de http://portal.corteconstitucional .gob.ec/Raiz/2014/004-14-SCN-CC/ REL \{_\}SENTENCIA__\}004-14-SCN-CC .pdf

Sentencia No. 0008-09-SAN-CC. (2009). Descargado de http: //portal.corteconstitucional .gob.ec/Raiz/2009/008-09-SAN-CC/ REL \{_\}SENTENCIA__\}008-09-SAN-CC .pdf

Sentencia No. 0731-10-EP. (2014). Descargado de https://www. corteconstitucional.gob .ec/images/stories/pdfs/Sentencias/ 0731-10-EP.pdf 
Solano, V. (2016). EL Neoconstitucionalismo. Una definición y una taxonomía latinoamericana. Ius Humani, 161-172.

Aceptado: 12 de octubre de 2018

Tarello, G. (2013). La interpretacion de la ley. Lima: Palestra.

Villanueva, R. (2015). La interpretación intercultural en el Estado constitucional. Revista de Derecho del Estado, 289-310.

Walsh, C. (2012). Interculturalidad y (de)colonialidad: Perspectivas críticas.

Zeta, V. V. (2007). Introduccion al Derecho Constitucional. Grijley E.I.R.L.

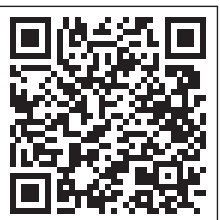

